\title{
Percepción de la calidad de vida referida por el paciente adulto con trasplante renal
}

\author{
María Isabel Durán Muñoz, Teresa Lope Andrea, María Rosario del Pino Jurado, María Cristina Chicharro \\ Chicharro, Elisa Matilla Villar
}

Enfermeras. Servicio de Nefrología del Hospital Clínico San Carlos. Madrid

\section{Resumen}

La percepción personal de un individuo de su situación de vida, dentro del contexto cultural y de valores en el que vive, y en relación con sus objetivos, expectativas, valores e intereses, se denomina, calidad de vida.

Como en estudios de pacientes crónicos, los factores más influyentes en la percepción de la calidad de vida, se clasifican en: paciente, medicamento, enfermedad y calidad de la interacción entre paciente y sistema sanitario.

\section{Objetivos:}

Describir la percepción que tienen los pacientes sobre su calidad de vida, e identificar factores que influyen en esta percepción.

\section{Material y métodos:}

Estudio descriptivo transversal en pacientes adultos, con trasplante renal, que acuden a nuestra consulta hospitalaria y son autosuficientes en el tratamiento.

Todos los pacientes han recibido charlas grupales sobre el manejo de su tratamiento antes del alta.

Recogida de datos con cuestionario anónimo autoadministrado: Escala validada: cuestionario de salud SF-12 (versión 2), durante 4 meses.

Se excluyeron pacientes que rechazaron participar y que necesitaban ayuda en el manejo del tratamiento. Se estimó un tamaño muestral de 222 pacientes. Variables dependientes: Calidad de vida. Variables independientes: Factores socio-demográficos, tipo inmunosupresores, educación sanitaria recibida por profesionales de la salud. Se solicitó consentimiento informado y se obtuvo informe favorable del Comité Ético de Investigación Clínica.

\author{
Correspondencia: \\ María Isabel Durán Muñoz \\ Servicio de Diálisis. Hospital Clínico San Carlos \\ C/ Profesor Martín Lagos s/n \\ 28040 Madrid \\ E-mail: iduran72@gmail.com
}

\section{Resultados:}

Participaron 209 pacientes. Edad media 55,2( $\pm 12,15)$ rango $21-80$ años, siendo el $61,8 \%$ hombres. El $42 \%$ eran jubilados, el 38,2\% tenían estudios secundarios, el $71 \%$ convivían en pareja.

El inmunosupresor más utilizado: Micofenolato de sodio (MYFORTIC@) $(65,5 \%)$ y el $46,7 \%$ tomaban dos inmunosupresores. La información sanitaria sobre efectos secundarios la consideran insuficiente un 20,5\%.

La media de la percepción de la calidad de vida es de $44,72 \pm 8,11$ para el componente físico y $47,47 \pm 8,8$ para el componente mental.

\section{Discusión:}

Los pacientes que llevan menos tiempo trasplantados refieren una mejor percepción de la calidad de vida.

Debemos tomar medidas de mejora en la educación sanitaria sobre efectos adversos.

Las charlas grupales informativas antes del alta del paciente creemos que han sido efectivas para la mejora del cumplimiento del tratamiento y en consecuencia para la mejora de su calidad de vida.

\section{PALABRAS CLAVE: \\ - TRASPLANTE RENAL \\ - CALIDAD DE VIDA \\ - EDUCACIÓN SANITARIA \\ - ENFERMERÍA}

\section{$\bullet \bullet \bullet \bullet \bullet \bullet \bullet \bullet \bullet \bullet \bullet \bullet \bullet \bullet \bullet \bullet \bullet \bullet \bullet \bullet \bullet$ \\ Perception of qualify of life reported by adult kidney transplant patients}

\section{Abstract}

The personal perception that an individual has of his or her situation in life, within the context of the culture and values in which he or she lives, and in relation to his/ her goals, expectations, values and interests is called quality of life.

As in studies with chronic patients, the factors that have the most influence on the perception of quality of life 
are classified as: patient, medication, disease and quality of interaction between patient and healthcare system. Objectives:

To describe the perception that patients have of their quality of life, and to identify factors that affect this perception.

\section{Material and methods:}

Transversal descriptive study of adult patients, with a kidney transplant, who come to our hospital outpatient clinic and are self-sufficient in treatment.

All patients received group talks on handling their treatment before being discharged.

Data collection using anonymous self-administered questionnaire: Validated scale: SF-12 health survey (version 2), for 4 months.

Patients who declined to participate and who needed help in handling the treatment were excluded. A sample size of 222 patients was estimated. Dependent variables: Quality of life. Independent variables: socio-demographic factors, type of immunosuppressive medication, health education received from healthcare professionals.

Informed consent was sought and a favourable report was obtained from the Clinical Research Ethical Committee.

\section{Results:}

209 patients participated. Average age 55.2( \pm 12.15$)$ range $21-80$ years, of whom $61.8 \%$ were men. $42 \%$ were retired, $38.2 \%$ had secondary school qualifications, $71 \%$ lived with a partner.

The most used immunosuppressive medication was mycophenolate sodium (MYFORTIC $\AA$ ) (65.5\%) and $46.7 \%$ took two immunosuppressive drugs. The information provided on side effects was considered insufficient by $20.5 \%$.

The average perception of quality of life is $44.72 \pm 8.11$ for the physical component and $47.47 \pm 8.8$ for the mental component.

\section{Discussion:}

Patients who have received their transplants more recently report a better perception of quality of life.

We should take measures to improve education on side effects.

We believe that the informative group talks before discharging patients have been effective in improving compliance with treatment and consequently in improving their quality of life.

\section{KEY WORDS:}

$\begin{array}{ll}- & \text { KIDNEY TRANSPLANT } \\ - & \text { QUALITY OF LIFE } \\ - & \text { HEALTH EDUCATION } \\ - & \text { NURSING }\end{array}$

\section{Introducción}

Según la Organización Mundial de la Salud, la percepción personal de un individuo de su situación de vida, dentro del contexto cultural y de valores en el que vive, y en relación con sus objetivos, expectativas, valores e intereses, se denomina, calidad de vida. Por tanto, abarca aspectos objetivos y subjetivos del propio individuo(1).

La percepción de la calidad de vida del paciente con trasplante renal está íntimamente ligada a la percepción que tenían dichos pacientes en su situación de insuficiencia renal crónica con tratamiento sustitutivo dialítico y en los efectos que la enfermedad produce sobre la vida diaria del paciente. Dichos pacientes, siguen siendo pacientes crónicos, con un alto índice de morbilidad, que precisan de un continuo control médico especializado y que están sometidos a una medicación inmunosupresora que genera efectos secundarios adversos.

La mejoría en la supervivencia de pacientes con trasplante renal, se debe al desarrollo de terapias inmunosupresoras orientadas a prevenir el rechazo del injerto pero el cumplimiento adecuado del tratamiento inmunosupresor es fundamental en este tipo de pacientes.

Como en otros estudios de pacientes con trastornos crónicos, los factores más destacados que influyen en la percepción de la calidad de vida se clasifican en: el propio paciente, el medicamento, la enfermedad y la calidad de la interacción entre el paciente y el sistema sanitario.

En España durante el año 2010 se hicieron 2225, de los cuales 420 se realizaron en Madrid. (www.ont.es). EI número de trasplantes renales realizados en este hospital desde sus inicios en el año 1980 es de 1740.

\section{Objetivo principal}

Conocer la percepción de la calidad de vida de pacientes adultos, mayores de 18 años, con trasplante renal funcionante en nuestro hospital.

\section{Objetivos secundarios}

Identificar factores que influyen en la percepción de la calidad de vida de los pacientes con trasplante renal (Socio-demográficos, medicamentos, educación sanitaria, calidad de vida).

\section{Material y métodos}

\section{Diseño y ámbito}

Estudio descriptivo a través de un corte transversal en pacientes adultos, mayores de 18 años, con injerto renal fun- 
cionante, que acuden a la consulta de trasplante renal, de ámbito hospitalario del Hospital Clínico San Carlos.

Todos los pacientes han recibido charlas grupales sobre el manejo de su tratamiento antes del alta.

La selección de pacientes se llevó a cabo de manera consecutiva y desde el comienzo del estudio, a todos los que acudían a la consulta y eran autosuficientes en el manejo de su tratamiento y deseaban participar en dicho estudio. Se entregó una hoja informativa sobre el estudio a realizar y se pidió consentimiento informado.

\section{Criterios de inclusión}

Pacientes con trasplante renal funcionante, adultos, mayores de 18 años, que acuden a la consulta de trasplante renal de ámbito hospitalario y son autosuficientes en el manejo de su tratamiento.

\section{Criterios de exclusión}

Se excluyeron del estudio aquellos pacientes que no quisieron participar libremente, no firmaron el consentimiento informado y los que necesitaban ayuda o no eran autónomos en el manejo de su medicación.

\section{Tamaño muestral}

El tamaño total de nuestra población es de 817 pacientes. En función del registro de trasplantes renales de la consulta de enfermería, el número de sujetos potenciales candidatos a participar en el estudio sería de 150 al mes. Se estimó que la mitad de esta muestra no cumpliría los criterios de inclusión y/o serían visitas consecutivas, por lo tanto sería necesario un periodo de estudio de cuatro meses (Junio-Septiembre) para reclutar el tamaño muestral que fue de 209 pacientes.

\section{Recogida de datos}

La recogida de datos consistió en la cumplimentación de un cuestionario auto-administrado, anónimo y no identificativo que se les facilitó a los pacientes y que conllevaba menos de 15-20 minutos en rellenarlo. La entrega de los cuestionarios se hizo en sobre cerrado y se recogieron en un buzón que dispusimos para ello.

\section{Herramienta de estudio}

Como herramienta de estudio se ha elaborado un cuestionario de 34 preguntas repartidas de la siguiente manera:

- 10 preguntas referentes a datos socio-demográficos del paciente.

- 7 preguntas referentes a la toma de medicación en general, tanto inmunosupresora como otras medicaciones para otras patologías asociadas.
- 6 preguntas referentes a la medicación inmunosupresora específica del trasplante.

- 4 preguntas referentes a la información recibida por el personal sanitario.

- En 7 preguntas, se utilizó el Cuestionario de salud SF12 (versión 2) [Anexo 1] $]^{(2)}$. Es una escala que proporciona un perfil del estado de salud de los pacientes. El cuestionario SF-12 es una versión reducida del cuestionario SF-36 ${ }^{(3)}$. Existen dos versiones del cuestionario SF-12, las modificaciones de la versión 2 permite: a) mejoras en instrucciones y presentación destinadas a facilitar la autoadministración y b) el número de respuestas. Consta de 12 ítems provenientes de las 8 dimensiones del SF-36. Las opciones de respuesta son de tipo Likert que evalúan intensidad o frecuencia. El número de opciones de respuesta oscila entre tres y cinco dependiendo del ítem. Para facilitar la interpretación estas puntuaciones se estandarizan con los valores de las normas poblacionales, de forma que 50 (desviación estándar de 10) es la media de la población general. Los valores superiores o inferiores a 50 deben interpretarse como mejores o peores, respectivamente que la población de referencia. Los valores que toma la escala tienen un recorrido desde 0 al00 puntos, siendo 0 el peor estado de salud y 100 el mejor estado de salud.

\section{Variables de estudio}

- Variables Dependientes

- Calidad de vida: Cuestionario de salud SF-12 (versión 2).

- Variables Independientes

- Factores socio-demográficos: Sexo, edad, nivel de estudios, situación laboral, situación de convivencia

- Medicación: medicación inmunosupresora y medicación para otras patologías.

- Educación sanitaria recibida por parte de los profesionales de la salud.

\section{Análisis estadístico}

Se diseñó una base de datos en Access 2003 en la que se recogió la información de las variables del cuestionario.

\section{Análisis descriptivo}

Las variables cualitativas se presentan con su distribución de frecuencias. Las variables cuantitativas se resumirán en su media y desviación estándar (DE). Las variables cuantitativas que no siguen una distribución normal se resumirán con la mediana y el rango intercuartílico (RIC).

\section{Análisis univariado}

Se evaluó la asociación entre variables cualitativas con el test de C2 o prueba exacta de Fisher, en caso de que 
más de un $25 \%$ de los esperados fueran menores de 5 . En el caso de variables ordinales se contrastó la hipótesis de tendencia ordinal de proporciones. Las comparaciones de medias entre dos grupos independientes se realizaron mediante la prueba $T$ de Student para grupos independientes. En el caso de variables que no se distribuyan de manera normal se utilizó el test no paramétrico de la mediana.

En todos los contrastes de hipótesis se rechazó la hipótesis nula con un error de tipo I o error a menor a 0.05. El paquete informático que se utilizó para el análisis fue el SPSS 15.0.

\section{Consideraciones éticas}

\section{Protección de los datos}

En ningún caso se han incluido en las bases de datos, dato alguno que pudiera directa o indirectamente identificar a ningún sujeto de modo individualizado. Con ello, se respetan las normas internacionales de protección de datos, así como la legislación española vigente. Los investigadores responsables son garantía de la seguridad de las bases de datos, que no serán utilizadas para otro fin que el señalado en el apartado de objetivos específicos.

\section{Consentimiento informado}

Se administró la hoja de consentimiento informado a los pacientes, previo a la realización del estudio, respetando las normas de la declaración de Helsinki. En él se explicaban los objetivos y procedimientos del estudio y se aseguraba la confidencialidad de los datos. Se recogió convenientemente firmada.

Informe favorable del comité ético de investigación clínica

\section{Resultados}

De 209 pacientes de los que se recibió el cuestionario, la edad media es de 55,25 $\pm 12,15$ (media \pm D.E.) de un rango comprendido entre $21-80$ años; de los que el $61,8 \%$ son hombres y el $37,8 \%$ son mujeres. El $38,2 \%$ de estos pacientes tienen un nivel de estudios secundarios, la situación de convivencia más frecuente es: con pareja y más familia un $40,6 \%$, seguido de vivir con pareja y sin familia con un $30,4 \%$, lo que nos lleva a deducir que el $71 \%$ de nuestros pacientes conviven con pareja. En cuanto a la situación laboral, la condición de jubilado es la más frecuente con un $42,0 \%$ y la menos frecuente es la de estudiante con un 1,9\%.
En cuanto a la medicación inmunosupresora que toman, el medicamento inmunosupresor, sólo o combinado, más utilizado entre nuestros pacientes es el Micofenolato de sodio (MYFORTIC $\AA$ ), con un $65,5 \%$, seguido del Tacrolimus (PROGRAF®), con un 56\%. El 46,7\% refieren que toman dos inmunosupresores.

En cuanto a la toma de medicación, la manera de recordar la toma de medicación en un $61,7 \%$, es de memoria y si tuvieran que evitar una de las tomas, preferirían la de la noche un $57,2 \%$, porque según refieren les permite organizar mejor su ocio y relaciones personales $(41 \%)$ y porque se adapta mejor a su vida familiar $(30,1 \%)$.

En cuanto a los efectos secundarios de los inmunosupresores, el $54,6 \%$ de los pacientes encuestados, refiere no tener ninguno; siendo los problemas dermatológicos con un $28,5 \%$, los más referidos, seguido de problemas musculo-esqueléticos con un $28,0 \%$ y gastrointestinales con un $25,5 \%$.

Nuestros pacientes, creen que la información recibida por parte de los profesionales sanitarios sobre los beneficios de la medicación es la adecuada en un $87,9 \%$. En cambio, sobre la información de los efectos adversos, un $20,5 \%$ opina que es insuficiente y sólo un $73,7 \%$ creen que es adecuada. La información sobre las horas a las que deben tomar la medicación, el $88,8 \%$ opina que es fácil de entender y el $96,6 \%$ refiere que siguen las instrucciones que le dieron los profesionales sanitarios.

La calidad de vida de nuestros pacientes, medida con la escala SF-12v2, nos da un componente físico y un componente mental. La puntuación media para el componente físico es $44,72 \pm 8,11$ y para el componente mental $47,47 \pm 8,80$.

En cuanto al componente físico, los de menor edad ( $\leq$ 45 años), tienen una mejor percepción de la calidad de vida $(47,27 \pm 6,3)$ frente a los $>46$ años $(44,03 \pm 8,52)$ $(p=0,039)$.No hay diferencia por sexos ni por la situación de convivencia. Sobre el nivel de estudios, tienen mejor percepción de la calidad de vida los pacientes que tienen estudios universitarios, con una media de 47,89 $\pm 6,22$, frente al resto de pacientes que presentan una media de 43,68 $\pm 8,39$ ( $p=0,003$ ). Los pacientes que están en activo, también tienen mejor percepción de la calidad de vida $(48,46 \pm 5,71)$ frente al resto que presentan una media de 43,46 $\pm 8,28(p=0,000)$.

En cuanto al componente mental, no presenta relación con la edad. Los hombres tienen una mejor percepción 
de la calidad de vida, con una media de 48,57 $\pm 8,52$ frente a las mujeres que presentan una media de 45,67 $\pm 9,03(p=0,031)$. Los pacientes que llevan menos tiempo trasplantados, también refieren una mejor percepción de la calidad de vida 48,76 $\pm 8,99$, frente a los que llevan más tiempo 46,06 $\pm 8,40(p=0,047)$.

\section{Discusión}

Estamos ante un grupo de pacientes cuya media de edad son 55 años y mayoritariamente están jubilados y con pareja. Coincidimos con el estudio publicado en $2006^{(4)}$, según el que los pacientes con nivel de estudios universitarios y que están en activo tienen mejor percepción de la calidad de vida.

Teniendo en consideración que la percepción de la calidad de vida del paciente con trasplante renal está íntimamente ligada a la percepción que tenían dichos pacientes, en su situación de insuficiencia renal crónica con tratamiento sustitutivo dialítico, no es extraño que los pacientes que llevan menos tiempo trasplantados, refieran una mejor percepción de la calidad de vida frente a los que Ilevan más tiempo, ya que tienen más presente su percepción de calidad de vida que cuando estaban sometidos a tratamiento dialítico. Después de ser trasplantados, los pacientes conceptualizan la calidad de vida como la cualidad de regresar a la vida normal, reincorporándose a actividades familiares, sociales, culturales, laborales, etc., las que antes no podían realizar por las complicaciones propias de la insuficiencia renal crónica ${ }^{(5)}$.

Consideramos necesario tomar medidas de mejora en cuanto a la información sanitaria sobre los efectos adversos de la medicación inmunosupresora, ya que nuestros pacientes la consideran insuficiente.

El personal de enfermería juega un importante papel en la educación sanitaria de nuestros pacientes con intervenciones enfermeras como el manejo de la medicación, asegurando así la mejora de la calidad de vida.

Las charlas grupales informativas antes del alta del paciente creemos que han sido efectivas para la mejora del cumplimiento del tratamiento y en consecuencia para la mejora de su calidad de vida.

Tras comprobar los resultados del trabajo, la enfermera de consulta de trasplante, sigue con la sistemática de las charlas grupales antes del alta del paciente, para completar la información que se les da a los pacientes durante el ingreso insistiendo especialmente en que hagan una puesta en común antes de marcharse a casa.

Los resultados nos sugieren realizar un nuevo estudio para autoevaluar la efectividad de estas sesiones informativas.

Recibido: 24 Mayo 2013

Revisado: 5 Junio 2013

Modificado: 11 Enero 2014

Aceptado: 27 Enero 2014

\section{Bibliografía}

1. Rodríguez Martín MC, Conejos Alegre C, Fuenmayor Díaz A, Mirada Ariet C, Sanz Izquierdo E, Torruella Barraquer C, Vela Ballestero A. Calidad de vida y trasplante renal en mayores de 65 años. Rev Soc Esp Enferm Nefrol 2009; ene-mar; 12(1).

2. Alonso J, Cols. Versión española de SF-12v2 $2^{\mathrm{TM}}$. Health Survey @. 1992,2002.

3. Alonso J, Regidor E, Barrio G, Prieto L, Rodríguez $C$ y de la Fuente $L$. Valores poblacionales de referencia de la versión española del Cuestionario de Salud SF-36. Med Clin Barc 1998; 111:410-416.

4. Valdés C, Ortega F. Avances en la calidad de vida relacionada con la salud y trasplante renal. Rev. Nefrología 2006; núm.2: 157-162.

5. Alarcón C, Aguilar 0, Jiménez A, Manrique C. Calidad de vida en pacientes con Trasplante renal medida a través de índice Karnofsky en un hospital general. Revista Medicina crítica y terapia intensiva. 2002; Vol.XVI, núm.4:119-123.

6. Ware JE Jr, Kosinski M, Keller SD. A 12-Item Short-Form Health Survey: construction of scales and preliminary tests of reliability and validity. Med Care.1996; 34:220-233. 
1. En general, usted diría que su salud es:
A. Excelente-(1)
B. Muy buena-(2)
C. Buena-(3)
D. Regular-(4)
E. Mala-(5)

2. Las siguientes preguntas se refieren a actividades o cosas que usted podría hacer en un día normal. Su salud actual, ¿le limita para hacer esas actividades o cosas? si es así, ¿cuánto?

A. Esfuerzos moderados, como mover una mesa, pasar la aspiradora, jugar a los bolos o caminarás de 1 hora.
a) Sí, me limita mucho-(1)
b) Sí, me limita un poco-(2)
c) No, no me limita nada-(3)

B. Subir varios pisos por la escalera
a) Sí, me limita mucho-(1)
b) Sí, me limita un poco-(2)
c) No, no me limita nada-(3)

3. Durante las 4 últimas semanas, ¿con que frecuencia ha tenido alguno de los siguientes problemas en su trabajo o en sus actividades cotidianas, a causa de su salud física?

A. ¿Hizo menos de lo que hubiera querido hacer?
a) Siempre-(1)
b) Casi siempre-(2)
c) Algunas veces-(3)
d) Nunca-(4)

B. ¿Tuvo que dejar de hacer algunas tareas en su trabajo 0 en sus actividades cotidianas?
a) Siempre-(1)
b) Casi siempre-(2)
c) Algunas veces-(3)
d) Sólo algunas veces-(4)
e) Nunca-(5)

4. Durante las 4 últimas semanas, ¿con qué frecuencia ha tenido alguno de los siguientes problemas en su trabajo o actividades cotidianas, a causa de algún problema emocional (como estar triste, deprimido, o nervioso)?

A. ¿Hizo menos de lo que hubiera querido hacer por algún problema emocional?
a) Siempre-(1)
b) Casi siempre-(2)
c) Algunas veces-(3)
d) Sólo algunas veces-(4)
e) Nunca-(5)

B. ¿Hizo su trabajo o sus actividades cotidianas menos cuidadosamente que de costumbre, por algún problema emocional?
a) Siempre-(1)
b) Casi siempre-(2)
c) Algunas veces-(3)
d) Sólo algunas veces-(4)
e) Nunca-(5)

5. Durante las 4 últimas semanas, ¿hasta qué punto el dolor le ha dificultado su trabajo habitual (incluido el trabajo fuera de casa y las tareas domésticas)?
A. Nada-(1)
B. Un poco-(2)
C. Regular-(3)
D. Bastante-(4)
E. Mucho-(5)

6. Las preguntas que siguen se refieren a como se ha sentido y como le han ido las cosas durante las 4 últimas semanas. En cada pregunta, responda lo que se parezca más a cómo se ha sentido usted. Durante las últimas 4 semanas ¿con que frecuencia....

A. ¿Se sintió calmado y tranquilo?
a) Siempre-(1)
b) Casi siempre-(2)
c) Algunas veces-(3)
d) Sólo algunas veces-(4)
e) Nunca-(5)

B. ¿Tuvo mucha energía?
a) Siempre-(1)
b) Casi siempre-(2)
c) Algunas veces-(3)
d) Sólo algunas veces-(4)
e) Nunca-(5)

C. ¿Se sintió desanimado y deprimido?
a) Siempre-(1)
b) Casi siempre-(2)
c) Algunas veces-(3)
d) Sólo algunas veces-(4)
e) Nunca-(5)

7. Durante las 4 últimas semanas, ¿con qué frecuencia la salud física o los problemas emocionales le han dificultado sus actividades sociales (como visitar a los amigos o familiares)
A. Siempre-(1)
B. Casi siempre-(2)
C. Algunas veces-(3)
D. Sólo algunas veces-(4)
E. Nunca-(5) 